\title{
Ruixia Pei's Experience in the Treatment of Hypothyroidism
}

\author{
Xingyu Chen'1, Fen Zhang1, Di Sun¹, Ruixia Pei ${ }^{2 *}$ \\ ${ }^{1}$ Shaanxi University of Chinese Medicine, Xianyang 712046, Shaanxi Province, China \\ ${ }^{2}$ Xi'an Hospital of Traditional Chinese Medicine, Xi'an 710021, Shaanxi Province, China \\ *Corresponding author: Ruixia Pei,29prx@163.com
}

\begin{abstract}
This study is to summarize Professor Pei's experience in treating hypothyroidism. Professor Pei has been engaged in clinical endocrinology for more than 30 years and has unique insights into hypothyroidism. She believes that the disease is caused by liver depression, phlegm binding, yin deficiency, and fire effulgence. The basic principles of treatment are regulating Qi movement and harmonizing viscera. During treatment, the emphasis is on the influence of emotions on the disease. Both the liver and spleen should be given equal attention and the medication should be mild with balanced cold and heat.
\end{abstract}

Keywords: Hypothyroidism; Case verification; Academic thought; Ruixia Pei

Publication date: September 2021; Online publication: September 30, 2021

\section{Introduction}

Hypothyroidism is a common clinical disease. It is characterized by an insidious onset and slow progress. The prevalence rate of the disease among women is often higher than that of men ${ }^{[1,2]}$. If women with hypothyroidism during pregnancy are not treated in time, there would be adverse effects on the mental and intellectual aspects of their babies ${ }^{[3-5]}$. In recent years, with the influence of the pace of life along with environmental factors, the incidence of hypothyroidism is increasing year by year. Studies have shown that the incidence of hypothyroidism in adults is $1.02 \%$ and the prevalence of subclinical hypothyroidism is $12.93 \%$, in which most patients with subclinical hypothyroidism have no obvious symptoms ${ }^{[6]}$. At present, Western medicine mainly emphasizes on lifelong thyroid hormone replacement therapy while traditional Chinese medicine has its unique advantages in relieving symptoms and improving indicators ${ }^{[7]}$. According to the clinical manifestations of hypothyroidism, traditional Chinese medicine divides hypothyroidism into "nontoxic goiter," "vacuity taxation," "edema," and other categories ${ }^{[8]}$.

Professor Ruixia Pei, a traditional Chinese medicine doctor in Shaanxi Province, has been engaged in clinical endocrinology, teaching, and scientific research for more than 30 years. She has rich clinical experience and profound theoretical attainments. She inherits the "harmonizing method" thought of Jingao Qin, attaches great importance to the liver and spleen during treatment, as well as the influence of negative emotions on the disease. The clinical effect is remarkable, which is deeply recognized by patients.

\section{Etiology and pathogenesis}

Professor Pei believes that the etiology and pathogenesis of hypothyroidism are closely related to emotions ${ }^{[9]}$. With the acceleration of urbanization, the pressures in people's lifestyles are gradually increasing, thus anxiety, tension, and depression are becoming more and more common. Imbalance emotions lead to liver 
dysfunctions, loss of its regulation function, depression, and transformation into fire, suffering from yin essence, condensing humor into phlegm, and blockage of the front neck. On the other hand, it can lead to the stagnation of liver-Qi, transverse invasion of the liver into the spleen, dysfunction of the spleen in transport, as well as phlegm dampness and internal resistance caused by a strong liver but weak spleen. The spleen is the source of acquired constitution; it affects the muscles of the limbs, and it is the source of the biochemistry of Qi and blood. Deficiency of spleen Qi as well as the insufficiency of Qi and blood result in limb weakness, shortage of qi, loss of strength, somnolence, deterioration of memory, alopecia, chills, and other symptoms.

\section{Principle of treatment}

During treatment, Professor Pei emphasizes on the treatment of liver and spleen. The drugs used are usually cold drugs, which are assisted by the restriction from warm drugs. Five flavors are concentrated in bitter, sweet, and spicy. Meridian tropism is mostly attributed to spleen meridian and liver meridian, thus soothing the liver and strengthening the spleen while treating according to different symptoms are the focus. Patients with blood stasis block are treated with blood circulation products, such as Rhizoma Ligustici and Curcumae Radix whereas patients with water-dampness and stagnation, drugs that fortify the spleen, percolate dampness, activate Qi, and eliminate phlegm are used, such as Poria, Rhizoma Pinelliae, Cortex Magnoliae Officinalis, etc. On the other hand, Rhizoma Dioscoreae, Corni Fructus, Radix Rehmanniae Preparata, and other drugs that nourish the kidneys and strengthen the essence are added in cases of longterm disease, endangering the kidneys.

\section{Example of a medical record}

The first visit to a doctor (or hospital) on March 15, 2021: Mr. X, male, 32 years old. The patient came to see a doctor with the chief complaint of abnormal thyroid function for 2 years and generalized fatigue for more than 1 month. The patient was found with abnormal thyroid function through examination 2 years ago (specific data is unknown) and was diagnosed with hypothyroidism after visiting a local hospital but the patient was not compliant. One month ago, due to reduced cognitive function and general fatigue, he was found in another hospital for a re-examination. The thyroid function test showed that his FT3 (free triiodothyronine) and FT4 (free thyroxine) were normal, but TSH (thyroid stimulating hormone) level was $6.36 \mathrm{mIU} / \mathrm{L}$ and TGAb (thyroglobulin antibodies) level was more than $1000 \mathrm{U} / \mathrm{mL}$. He was treated with oral levothyroxine sodium tablet (Euthyrox) 25ug, once a day. His current symptoms included tiredness, poor spirit, impaired memory, and daytime somnolence but he had difficulty to fall asleep at night. His intake of food was regular with normal defecation. His tongue was red with few mosses, and his pulse was thin. The TCM diagnosis was nontoxic goiter, deficiency, syndrome differentiation for hyperactivity of heart-liver fire, deficiency of both Qi and yin. The treatment was to clear the liver and reduce fire, nourish yin, the heart, and qi to invigorate the spleen. The formula was to add and subtract Minor Bupleurum Decoction combined with Pulse-Engendering Powder. The specific drugs are as follows: Radix Glehniae, Radix Ophiopogonis, Radix Curcumae vinegar 15 g, Bupleurum chinense vinegar, Rhizome Pinelliae Preparata, Cortex Magnoliae Officinalis, Rhizoma Ligustici, mix-fried licorice $10 \mathrm{~g}$, Schisandra chinensis vinegar $6 \mathrm{~g}$, stir-fried bitter orange $12 \mathrm{~g}$, and Radix Scrophulariae $20 \mathrm{~g}$. Six doses were given, decocted in water, 1 dose a day, taken in the morning and evening after meals. He was advised to regulate his emotions, work and rest regularly, as well as abstain from seafood, high iodine food, spicy irritants, and fried food.

The second visit on March 22, 2020: The patient mentioned that his palpitations significantly improved along with his daytime sleepiness and night rest. Otherwise, his food intake was good, and defecation was normal. His tongue was red with white moss and his pulse was thin. Radix Scrophulariae and Rhizoma 
Ligustici were removed from the prescription, but $6 \mathrm{~g}$ of Herba Menthae and $15 \mathrm{~g}$ of wine Cornus were added. Similarly, 6 doses were given, and the decocting method was the same.

The third visit on March 29, 2021: The patient had no obvious palpitations after taking the medication but was slightly sleepy. Otherwise, his food intake was good, he was able to rest at night, and had normal defecation. His tongue was red with white and thick moss, and his pulse was thin. Radix Rehmanniae Preparata and wine Cornus were removed from the prescription, but $30 \mathrm{~g}$ of Radix Trichosanthis and $15 \mathrm{~g}$ of Radix Rehmanniae Recens were added. The decocting method was the same as before but this time, 12 doses were given.

In his subsequent follow-up visits, the above prescriptions were the basic prescriptions and were modified according to evidence. Until his visit on June 19, the patient had no obvious discomfort and his TSH level had returned to normal. The patient was instructed to stop his medication for observation while reviewing his thyroid function regularly, improving his mood, and adjusting his diet. He was reminded to come for follow-up if he feels unwell.

\section{Conclusion}

In summary, Professor Pei emphasizes on the influence of emotions on diseases. Based on the principle of regulating the functioning of Qi and harmonizing viscera, she attaches equal importance in soothing the liver, regulating Qi, strengthening the spleen, and tonifying deficiency. The medication used is gentle with barely any harsh products and the dosage is light in addition to a balanced cold and heat.

\section{Disclosure statement}

The authors declare that there is no conflict of interest.

\section{References}

[1] Chinese Medical Association -Endocrinology Branch, 2007, Guidelines for Diagnosis and Treatment of Thyroid Diseases - Hypothyroidism. Chinese Journal of Internal Medicine, 46(11): 967-971.

[2] Chinese Endocrine Society CMA, 2017, Guidelines for Diagnosis and Treatment of Hypothyroidism in Adults. Chinese Journal of Endocrinology and Metabolism, 33(02): 167-180.

[3] Young J, Savoy C, Colman I, et al., 2020, Psychiatric Disorders in the Adolescent Offspring of Mothers with Thyroid Problems During Pregnancy. Child Psychiatry Hum Dev, 51(3): 461-470.

[4] Morreale de Escobar G, Obregon MJ, Escobar del Rey F, 2004, Role of Thyroid Hormone During Early Brain Development. Eur J Endocrinol, 151(3): U25-37.

[5] Xue G, Yan L, Li L, 2020, Research Progress on the Effect of Mild Hypothyroidism during Pregnancy on the Long-term Intelligence of Offspring. Chinese General Practice, 23(15): 18381842.

[6] Zhou Y, Wu JD, 2021, Research Progress in the Pathogenesis, Diagnosis and Treatment of Subclinical Hypothyroidism. Medical Recapitulate, 07: 1369-1372, 1379.

[7] Li QL, Liu HP, Wang YQ, et al., 2020, Discussion on Drug Use Rules of Traditional Chinese Medicine Differentiation and Treatment of Hypothyroidism in Recent 10 Years Based on Data Mining. Journal of Yunnan University of Traditional Chinese Medicine, 06: 79-84.

[8] Zhang MX, Ni Q, 2018, Progress in Traditional Chinese Medicine Treatment of Hypothyroidism. Beijing Journal of Traditional Chinese Medicine, 37(09): 851-854. 
[9] Zhang JL, Zhang ZQ, Hao F, et al., 2019, Examples of Pei Ruixia's Traditional Chinese Medicine Treatment of Subclinical Hypothyroidism. World Latest Medicine Information, 19(99): 265-267. 